\title{
Aprender a manejar los conflictos en las clases de educación física a partir del juego deportivo: un modelo de enseñanza para la comprensión Learning to manage conflicts in physical education classes from the sports tactical game: a teaching model for understanding
}

\author{
Robert Cebolla-Baldoví, Laura García-Raga \\ Universidad de Valencia (España)
}

\begin{abstract}
Resumen. La literatura científica constata la potencialidad que el conflicto puede generar en las aulas cuando se supera su sentido negativo y se inserta en el proceso educativo. Este planteamiento positivo del conflicto debiera ser asumido por los diferentes educadores de cualquier institución escolar para implementar programas alternativos a la violencia y enseñar a manejar los conflictos constructivamente. La Educación Física (EF) se presenta insólita para impulsar la gestión del conflicto en un espacio real de práctica y sobre un contexto de juego con equipos atacantes y defensores. Desde esta perspectiva, el objetivo principal del texto es mostrar una propuesta de enseñanza destinada a los últimos cursos de educación primaria. El desarrollo de la investigación, continuado en forma de tesis doctoral, se enmarca en un enfoque cuasiexperimental sobre un diseño de dos grupos (experimental y de control), desde una muestra de 130 participantes de dos colegios de Cullera (Valencia). El tratamiento (programa) se diseña desde la propia táctica del juego, y en su estructura queda incorporado el 'conflicto colateral', como fin didáctico, para aprender a gestionarlo entre sus miembros participantes. Sobre la práctica educativa, cabe reflejar que toma su origen de la enseñanza comprensiva; se fundamenta sobre un enfoque organizativamente participativo-colaborativo; y queda integrada en un escenario de cultura mediadora. Desde el ámbito de la EF escolar, se concluye que el método táctico-deportivo podría ser una alternativa para educar la gestión positiva del conflicto, posibilitando que el alumnado enfrentara las barreras de la comunicación y aprendiera a contrarrestar sus estereotipos destructivos.

Palabras clave: educación física escolar, diseño curricular, educación primaria, juegos de invasión, colaboración-oposición, conflicto, aprendizaje negociador, diálogo.
\end{abstract}

\begin{abstract}
Scientific literature confirms the potential that conflict can generate in the classroom when its negative meaning is overcome and it is inserted into the educational process. This positive perspective to conflict should be assumed by the different educators of any school institution to implement alternative programs to violence and teach how to handle conflicts constructively. Physical Education (PE) is unusual to promote conflict management in a real practical space and in a game context with attacking and defending teams. From this point of view, the main goal of the text is to show a teaching proposal aimed at the last years of primary education. The research study, continued in the form of a doctoral thesis, is framed in a quasiexperimental method on a two-group design (experimental and control), from a sample of 130 participants from two schools in Cullera (Valencia). The treatment (program) is designed from the game's own tactic, and the 'collateral conflict' is incorporated into its structure, as a didactic purpose, to learn to manage it among its participating members. Regarding educational practice, it should be reflected that it takes its origin from comprehensive teaching; it is based on an organizational participatory-collaborative strategy; and is integrated into a setting of mediating culture. In the PE context, it is concluded that the tactical-sports method could be an alternative to educate positive conflict management, enabling students to face communication barriers and learn to counteract their destructive stereotypes.
\end{abstract}

Keywords: school physical education, curriculum design, primary education, invasion games, collaboration-opposition, conflict, negotiator learning, dialogue.

\section{Introducción}

A finales del siglo pasado y principios del siglo XXI, el conflicto empieza a ser objeto de estudio en la especificidad de la doctrina pedagógica y la organización docente (Boqué, 2003; Funes, 2000; Jares, 1997; Tuvilla,

Fecha recepción: 23-01-21. Fecha de aceptación: 06-04-21

Robert Cebolla Baldovi

rocebal@gmail.com
2004). En base a la aceptación del conflicto, se genera interés por identificar la estructura social (percibida y oculta) de cada contexto escolar, junto a la preocupación por comprender su coexistencia de relaciones. A partir de ese momento, con el informe a la UNESCO, surge el desafío de encarar la rígida tradición del siglo pasado y encontrar formas no violentas de resolver los desacuerdos (Delors, 1996).

En cuanto a qué se entiende por conflicto, Rozemblum (1998) apunta que «es parte natural de 
nuestra vida; no es ni bueno ni malo, simplemente existe» (p. 77). Para Hocker y Wilmot (1985), el conflicto es la «lucha ejercitada entre al menos dos personas o grupos interdependientes que perciben objetivos incompatibles, recompensas escasas e interferencias del otro para lograr sus metas» (p. 23).

Desde una perspectiva renovadora, se subraya que el principal reto de la educación debe ser «convertir a los centros en espacios de convivencia» capaces de «conformar sociedades vertebradas y cohesionadas» (GarcíaRaga \& López-Martín, 2010, p. 22) y en donde se gestionen los conflictos de manera positiva para transformarlos en oportunidades de aprendizaje. Leiva (2011) aclara que los centros educativos que pretenden ser inclusivos necesitan aceptar y reconocer que «el conflicto va a ser algo natural en estos nuevos escenarios educativos ricos, complejos y diversos» (p. 372).

Para gestionar los conflictos de manera positiva, se incorpora la mediación, que consideramos se distingue del modelo 'autoritario-punitivo' y del 'comunitario o dialógico' por no tratar de eliminar los conflictos o de evitar «solucionarlos desde la propia comunidad [educativa] mucho antes de que aparezcan» (Flecha \& García, 2007, p. 74). El modelo mediador se vincula al «proceso de gestión pacífica de conflictos» (Boqué, 2018, p. 7) desde la oportunidad de examinar «la expresión de necesidades mal resueltas» (Boqué, 2020, p. 177) y que sale a la luz cuando se concede dar voz a los afectados y se descubren las causas del conflicto. En la práctica, este modelo permite al estudiantado «elaborar soluciones colaborativas y participativas en interacción con los componentes clave de todo conflicto», es decir, personas, problemas y procesos (Hamilton, 2015, p. 4). El modelo mediador se diferencia del modelo comunitario en la medida que acepta la presencia del conflicto como contenido educativo. El conflicto, específicamente, no es abordado desde la aplicación del «modelo experto» (Flecha y García, 2007, p. 73) por parte de alguien experimentado y ajeno al conflicto ocasionado, sino más bien desde la cercanía del 'profesorado dinamizador-regulador' y la incorporación del 'alumnado ayudante' (Torrego, 2013) o la 'mediación entre iguales'.

Por otro lado, el enfoque de acción impulsado por la mediación entre iguales va más allá de un planteamiento preventivo con significado de contención del conflicto, al contrario, contempla gestionar la situación conflictiva de la mejor manera posible e insertándola en el trabajo educativo y curricular de la organización escolar (Aguilar \& Castañón, 2013; Boqué, 2007; Fuquen, 2003; Leiva, 2008; Munduate, Ganaza \& Alcaide, 1993;
Sánchez, 2018; Villalba, 2016). En último término, diversos autores plantean no prevenir el conflicto, pero sí justifican la prevención de la violencia. Con mayor precisión, cabe reconocer que no defienden oponerse al conflicto; ni lo consideran disfuncional en sí mismo, ni tratan de prevenirlo (evitando, ocultando o eliminándolo). Básicamente, para Bercovitch, Kremenyuk y Zartman (2009) «la noción de que el conflicto pueda ser eliminado es idealista e irreal» (p. 3). Llegados a este punto, la mediación entre iguales se evidencia como un marco apropiado para ejercitar las habilidades de comunicación adquiridas en pro de resolver eficazmente futuros conflictos del mundo real (Dummer, 2010; Harris, 2005; Whiteside, 2016).

De igual modo, el modelo mediador se esfuerza en promover «la colaboración y el valor de las relaciones» (Burton, 1990, p.V) a fin de permitir tomar decisiones, traspasando acciones de poder y resolviendo los problemas a satisfacción de todas las partes (Burton, 1997, pp. 3-4). Boqué (2007) recalca que el neologismo «provención» creado por Burton, «remite a una actuación temprana sobre el entorno que no teme analizar e identificar aquellos elementos que generan insatisfacción, desigualdad, frustración, abuso, exclusión o falta de recursos, y promueve condiciones más justas e igualitarias» (p. 60). Y, en consonancia, podríamos decir que existe un paralelismo entre el modelo mediador y el comunitario (en su perspectiva crítica) en cuanto que, ambos, comparten la aplicación de procesos de diálogo, originados desde prácticas educativas democráticas y, cuya meta, trata de hacer frente a los obstáculos que generan desigualdades sociales (Aubert, Duque, Fisas \& Valls, 2004). Los dos modelos facilitan espacios y momentos para tomar parte y encontrar acuerdos conjuntos. Si bien, es cierto que el «modelo dialógico» involucra a toda la comunidad educativa para «asegurar que todas las normas o propuestas que se decidan sigan un procedimiento dialógico» y solo se acepten «las pretensiones de validez y no las de poder» (Flecha y García, 2007, p.74). A tal efecto, los modelos mediadores pudieran dificultar la participación real de la comunidad educativa si no se la toma en consideración para adherirse a un modélico plan integrado de mejora de la convivencia. Completando lo anterior, Torrego (2006), según su descripción de los diferentes modelos de respuesta a los conflictos (punitivo, relacional e integrado), especifica que el plan integrado «plantea una exigencia global con grandes implicaciones organizativas dentro de la estructura general, una formación concreta para profesores y alumnado basada en los principios 
educativos del diálogo, y una participación activa»; además, recalca que «se hace imprescindible reflexionar» por parte de la comunidad educativa, explicitando los valores sobre los que se quiere educar y qué estructuras son necesarias para garantizar el desarrollo de la organización (pp. 29-36).

En materia de los programas de mediación entre iguales, empiezan a implementarse a finales de los sesenta del siglo pasado en los Estados Unidos de América y se introducen en España a mediados de la década de los noventa. Pinto y Oliveira (2020) identifican que la mediación escolar enmarcada como «estrategia de intervención socioeducativa», se ha ganado «la atención de las escuelas, así como de la academia que busca reconocer sus ventajas y potencialidades a nivel personal, interpersonal y organizacional» (p. 114). García-Raga, Boqué y Grau (2019) precisan que «el sentido pedagógico de la mediación podría sintetizarse en tres fines: la resolución de conflictos, la prevención de la violencia y el fortalecimiento personal» (p. 106). Desde esta triple finalidad, el modelo mediador se proyecta sobre diferentes objetivos: para comenzar, Boqué (2018) nos indica que la mediación escolar toma una meta educativa no solamente para quienes participan del programa (individualidades en conflicto) sino también para el grupo-clase en su participación por la búsqueda de soluciones. Es más, los centros educativos que aumentan su comprensión del conflicto, también se benefician cuando detectan áreas factibles de mejora. Seguidamente, diferentes autores corroboran la potencialidad que la mediación genera no solo en la labor de transformar los conflictos escolares (corto plazo), sino también en el aprendizaje y el desarrollo-adquisición de competencias básicas (medio plazo); así como la ayuda que ejerce sobre el crecimiento del factor de cohesión intragrupal y la oportunidad que, a largo plazo, ofrece al conjunto de la sociedad como dinamizador de procesos de cambio (Adigüzel, 2015; Condette, 2020; Miranzo, 2018; Viana \& López, 2015). Sobre los principios de la mediación se enumeran los más importantes: prevención, voluntariedad, confidencialidad, libre decisión, empoderamiento (Boqué, 2018); concepción positiva del conflicto, diálogo como respuesta constructiva, contextos cooperativos, habilidades de autorregulación y autocontrol, actitudes de apertura, comprensión y empatía (Sanchez, 2016); aplicar la escucha activa, otorgar confianza en las posibilidades de éxito, tener paciencia, replantear el conflicto, sugerir posibilidades de resolución en caso de estancamiento (Jares, 2001).

Respecto a la forma de implementar la ayuda me- diadora por parte del profesorado o alumnado, se señala como aconsejable que se trabaje en equipo y se actúe en parejas (co-mediación) para «facilitar captar diferentes percepciones del conflicto, permitir un mayor control del proceso de mediación y dotar de tranquilidad a los propios mediadores» (Martínez, Sahuquillo \& GarcíaRaga, 2013, p. 63). En cuanto a la implantación efectiva de un programa mediador, Ortega-Ruiz y Del Rey (2006) admiten que debería conseguir que el alumnado obtuviera un control progresivo de «habilidades de comunicación, diálogo y comprensión emocional para así desarrollar poco a poco las competencias necesarias para resolver por sí solos los conflictos» (p. 3).

Todas las ventajas señaladas nos motivan a diseñar una propuesta educativa que aspire a impulsar la cultura de la mediación en un centro escolar en el que se constata un claro predominio del conflicto en los últimos cursos de primaria, detectándose la falta de habilidades empáticas, impulsividad, incapacidad de escucharse o comunicación a gritos y, en consecuencia, contestaciones reactivas como el uso de la fuerza. Esta iniciativa pedagógica se fundamenta desde la potencialidad táctica del juego deportivo, y se presenta como posible herramienta para equilibrar la conflictiva oposición del juego sociomotriz. Canales, Aravena, CarcamoOyarzun, Lorca y Martinez-Salazar (2018) afirman que «el juego y el deporte son importantes herramientas para fomentar diálogos simétricos y relaciones positivas en función de objetivos y metas compartidas» (p. 216). Asimismo, Medina y Reverte (2019) amplían que desde el contexto de la práctica deportiva, los jugadores «se ven expuestos a una enorme cantidad de situaciones donde es necesaria la resolución de conflictos» $\mathrm{y}$ que, además, se presentan como «idóneas para trabajar hacia la prevención de la violencia escolar» (p. 59).

En este punto, se hace necesario introducir el Modelo Pedagógico Comprensivo de iniciación deportiva, como apropiado para la gestión del conflicto y que conceptualiza nuestra práctica educativa. Esta metodología sobrepasa un modelo de instrucción directa (alumnado receptor y aprendizaje memorístico) y evoluciona de un paradigma conductista a otro cognitivista, convirtiéndose en un «enfoque más dinámico y adaptado a las necesidades de aprendizaje» del educando (Martín-Barrero \& Camacho, 2020). Cabe añadir que, esta forma de aprender, sobrepasa un estilo de enseñanza altamente estructurado por el profesorado, siendo el paradigma de 'aprendizaje colaborativo' el más adecuado para responsabilizar al propio aprendiz (Zañartu, 2003). Debido a la interactividad constituida entre el 
alumnado, se generan «actividades extras» como «explicaciones, desacuerdos y regulación mutua»; provocando, por regla general, «mecanismos cognitivos adicionales» (internalización, extracción); y que, después de todo es lo que nos ofrece la posibilidad de aprender (Zañartu, 2003, p. 4).

Desde el «Teaching Games for Understanding» (SánchezGómez, Devís-Devís \& Navarro-Adelantado, 2014) o la pedagogía no lineal, se «intenta que los jugadores puedan dar solución a los diferentes problemas con distintas soluciones» (Cantos \& Moreno, 2019, p. 403). Según Castejón y Díaz (2011), «el planteamiento comprensivo implica que el diseño de las tareas debe situar al alumnado en contextos de práctica cercanos a los del juego real, de manera que provoquen situaciones-problema con un carácter prioritariamente táctico» (p. 34). Este aprendizaje comparte una perspectiva constructivista, buscando más allá del saber procedimental (ejecutar y tomar buenas decisiones tácticas) y desarrollando el conocimiento conceptual cuando se trata de comprender «lo que se hace y por qué se hace» (López-Ros, Castejón-Oliva, Bouthier \& Llobet-Martí, 2015, p. 54). De forma explícita, también queremos resaltar las posibilidades de aprendizaje que mantienen los juegos modificados en intersección con la presencia del conflicto. Compartimos con el profesor e investigador Devís-Devís (1996) que los «juegos de invasión» pueden ser buena opción tanto para que el profesorado enseñe los principios tácticos de 'colaboraciónoposición’ como para que el alumnado se implique activamente en su aprendizaje (p. 54).

En fin, el objetivo del texto es mostrar un diseño didáctico que pueda ser relevante para aprender a gestionar el conflicto desde el dinamismo complejo del juego deportivo.

\section{Enfoque y metodología}

La propuesta presentada se enmarca en un enfoque cuasiexperimental, utiliza metodología mixta y se inserta en un estudio mucho más amplio en forma de tesis doctoral. El propósito final de la investigación en conjunto será examinar el impacto que podrá tener la aplicación de un programa integrado de mejora de la convivencia. Para mayor detalle, se describen las hipó- tesis específicas sostenidas en esta investigación y que se asocian al programa táctico-deportivo planteado (figura 1): (1) «Las estrategias de intervención educativa impulsarán un cambio comprensivo y democrático en el estilo de afrontamiento del conflicto, posibilitando diálogo autorregulador de la escalada emocional (enfado-ira)»; (2) «El programa de cultura de mediación ofrecerá una eficiente intervención reeducadora, equilibrando y compensando estilos unilaterales (ganar o perder), y generando diálogo respetuoso (ganar-ganar)»; (3) «La seriedad del juego ligada a la motivación e intransigencia del propio participante posibilitarán, en momentos de enfrentamiento, un contexto dinámico y eficaz para la creación de aprendizaje» (Cebolla-Baldoví, 2021, p. 16).

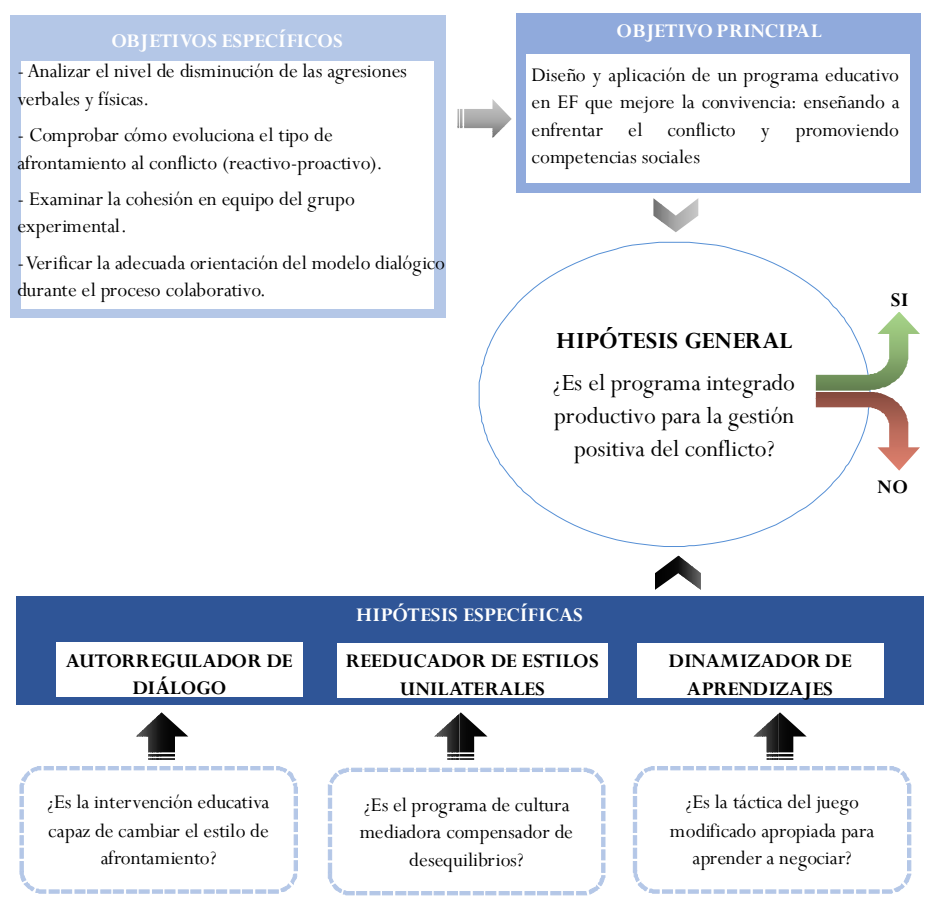

La presente investigación empleará un diseño de dos grupos no equivalentes (grupo experimental y grupo de control) vinculados a dos escuelas públicas de la localidad de Cullera (Valencia). La muestra agrupará a 65 participantes de cuarto, quinto y sexto cursos de primaria en cada colegio. Principalmente, el alumnado pertenece a un nivel sociocultural y familiar medio, con un 23\% de inmigrantes extranjeros (países del Este de Europa y Latinoamérica) y nacionales de procedencia castellana; además de hallarse la situación de desventaja social por escasez de recursos. El grupo experimental correspondiente al centro del maestro investigador será expuesto a la influencia del programa pedagógico; y al grupo de control (otro colegio con similares características) se le mantendrá libre de cualquier 
tratamiento. Igualmente, ambos grupos serán sometidos a un análisis pretest y postest, lo que permitirá comparar estadísticamente las medidas postest de la variable dependiente en ambos grupos (ver tabla 1).

Tabla 1.

\begin{tabular}{|c|c|c|c|c|c|}
\hline Grupos & Sujetos & Asignación & Pretest & Tratamiento (VI) & Postest (VD) \\
\hline Control & 65 & No azar & Sí & 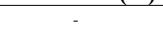 & Sí \\
\hline Experimental & 65 & No azar & Sí & Programa & Sí \\
\hline
\end{tabular}

Las técnicas e instrumentos utilizados para la recogida de información serán la observación (participante y sistemática) por parte del maestro investigador, las entrevistas y los grupos de discusión llevados a cabo durante las sesiones de clase y/o posteriormente. Por otra parte, desde la perspectiva cuantitativa, se buscará explicar la relación causa-efecto y comprobar la verificación de las hipótesis a través de test, pruebas objetivas y cuestionario. Cabe añadir que para llevar a cabo el procedimiento se contempla la obtención de permisos por ambos centros educativos y de los familiares del alumnado participante; junto con la validación del cuestionario (tabla 2), estimando la validez (juicio de expertos) y pasando la prueba piloto a 35 alumnos para comprobar el grado de fiabilidad alfa de Cronbach $(\geq 0,7)$.

\section{Fundamentación de la propuesta}

\section{Contexto de la EF}

Centrándonos en la EF, se puede verificar la existencia de una reciente literatura científica que la concibe como el espacio óptimo para la presencia y transformación de los conflictos. En general, las fuentes del conflicto están motivadas por discrepancias en el procedimiento, de naturaleza afectiva, por percepciones o intereses contrapuestos (Ruiz, 2008). Por ello, se hace necesario que los participantes aprendan a «gestionar sus emociones, así como a mejorar sus habilidades sociales» (Hernández, Morán \& Bucheli, 2018, p. 162); además de adquirir competencias para enfrentar «el sentirse ofendido, la envidia, las peleas y el enojo» (Bello-Dávila, Rionda-Sánchez \& Rodríguez-Pérez, 2010, p. 41). Básicamente, la EF se contempla como una área curricular vivencial apta para el correcto e íntegro desarrollo de la educación emocional y del aprendizaje de habilidades prosociales (Alcaraz-Muñoz, Alonso \& Yuste, 2017; Cantón \& Leon, 2005; Capllonch, Figueras \& Lleixà, 2014; Jaqueira, Lavega-Burgues, Lagardera \& Rodrigues, 2014; Monzonís \& Capllonch, 2014; Sáez \& Lavega-Burgués, 2015; Sáez, Lavega-Burgués, Lagardera, Costes \& Serna, 2014; Sáez, Lavega-Burgues \& March-Janés, 2013).

En el ámbito del juego, Sáez y Lavega-Burgues (2014) manifiestan que el profesorado de EF «dependiendo del tipo de situaciones motrices que propone, induce a sus alumnos a relacionarse entre ellos de una manera determinada» (p. 50). Explícitamente, en función del tipo de juego elegido y la lógica interna de la acción motriz, se identifican unas relaciones motrices concretas; $y$, consiguientemente, pueden provocar procesos de conflicto: primero, manifestado por el agente generador (jugador participante); y, segundo, replicado por la reacción al estímulo inicial (por 
parte del jugador adversario u otro jugador del equipo aliado). Sáez (2011) distingue que dicho conflicto motor «implica siempre una respuesta en forma de agresión verbal, física o mixta» (p. 119); provocando, posteriormente, «una intervención por parte de los protagonistas y/o el profesor» y ocasionando diversas actuaciones según la categoría de acción-reacción del conflicto (p. 120).

\section{Modelos de gestión de conflictos en EF}

Fruto de nuestra revisión, se establecen tres bloques de autores según el modo de entender el conflicto:

Excluyen el conflicto. Programas originados desde modelos comunitarios o dialógicos, preventivos y que consideran el conflicto como un acontecimiento negativo que se debe erradicar (Buscà, Ruiz \& Rekalde, 2014; Capllonch, Figueras \& Castro, 2018; Martín \& Ríos, 2014; Monzonís \& Capllonch, 2014).

- Contrarrestan el conflicto. Desde otro criterio, existen programas que acogen el juego de oposición en equipo para desarrollar el aprendizaje de las relaciones interpersonales y con el objeto de minimizar las acciones violentas: mediante la identificación de los conflictos del propio juego y su posterior modificación de reglas (lógica interna) o cambio de juego si no fuera efectivo (Sáezet al., 2014, 2013). Otros planteamientos desean combatir los conflictos a través de la incorporación de juegos alternativos y motivadores como el kinball, baloncolí, indiaca, gimnasia en trampolín o bosaball (Ardila, Jaimes, Noy, Reina \& Martínez, 2019); al igual que implantan juegos de menor competitividad (artes marciales y los juegos populares) para regular las conductas violentas (Medina \& Reverte, 2019).

- Integran el conflicto. Opuesto a los modelos anteriores, se destaca un programa anual de intervención deportiva escolar (con ocho unidades didácticas) que acoge el conflicto para desarrollar las habilidades sociales. Entre sus resultados, la mediación, como procedimiento de resolución de conflictos, ayuda a que el alumnado utilice el diálogo, las actitudes de respeto y la solicitud de perdón ante los propios errores (Cepero, Marín \& Torres, 2010). Cabe anunciar la técnica 'role playing' (Cantón \& Leon, 2005) como facilitadora de la resolución de conflictos en la práctica deportiva escolar: (paso 1) a partir de un conflicto producido durante el juego (registrado por el participante de modo narrativo); (2) se debate el conflicto en grupo (describir cual habría sido la mejor forma de resolverlo teniendo en cuenta las actitudes trabajadas); (3) se representa el con- flicto (dramatización en equipos) junto a su acuerdo de solución; (4) se extrae un lema representativo de la resolución del conflicto aportada por el grupo-clase. Ruiz (2008) aplica un programa de tres meses de duración y trabajando actividades específicas en EF durante 10-15 minutos semanales, en el que elabora un mapa de actuación para cuando surge el conflicto: (paso 1) «nos tranquilizamos»; (2) «explico con calma lo que pienso y siento»; (3) «y escucho a mi compañero/a»; (4) «buscamos soluciones»; (5) «llegamos a un acuerdo»; (6) «ponemos en práctica el acuerdo»; (7) «vemos si el acuerdo ha sido bueno para todos» (p. 112). Esta modalidad es completada posteriormente con la formación del alumnado en mediación (pp. 102-103). Cerrando este bloque, Velázquez-Callado (2008) propone juegos cooperativos como medio para ofrecer herramientas al alumnado en el tratamiento del conflicto: (asunto 1) «el error está permitido; (2) se reflexiona sobre «qué es lo que ha pasado y por qué»; (3) se adquieren estrategias de grupo, traspasando «modos de actuar y de pensar» individuales o competitivos; (4) se consiguen habilidades para «alcanzar soluciones intermedias que permitan avanzar a todos» (pp. 145-155).

Tras explorar los modelos de gestión, seguimos considerando que el conflicto debe integrarse en la enseñanza del juego deportivo con el fin de aprender a manejarlo. Desde la especificidad práctica de la EF, se ha identificado una limitada publicación en cuanto a programas de acción mediadora. En todo caso, en el ámbito general de la educación escolar, sí que existen destacadas intervenciones de cultura mediadora aplicadas sobre las aulas (Barruel \& Nissanka, 2018; Gellin, 2018; Ibarrola-García \& Iriarte, 2013; Iglesias \& Ortuño, 2018; Samy, 2019; Silva \& Torrego, 2017). No nos queda duda que los programas de mediación entre iguales establecidos en otros espacios de aprendizaje (patio, clases), también pueden tener una clara transferencia de aplicación sobre el contexto de enseñanza de la EF.

\section{Planteamiento didáctico}

\section{Descripción del proceso formativo}

Nuestra propuesta va dirigida a los cursos de cuarto, quinto y sexto de primaria, e incorpora una formación de 27 sesiones distribuidas a lo largo de los dos primeros trimestres del curso escolar, a razón de dos sesiones por semana. Este planteamiento curricular, constituido como 'bloque de juegos y actividades deportivas', se trabaja de forma paralela a la temporalización y enseñanza de otros contenidos como la 'actividad física y sa- 
lud', las 'habilidades motrices y coordinación', el 'conocimiento corporal y la autonomía' o la 'expresión motriz y comunicación' (desarrollados, frecuentemente, de forma globalizada durante la sesión). Respecto de los juegos concretados para cada sesión, se desarrollan los mismos para cada curso, aun cuando sea necesario establecer una progresión de menor a mayor dificultad. Aparte, se requiere contemplar las ideas previas del alumnado y la estructura interna de cada juego (número de elementos que dificultan) para decidir si se precisa añadir variantes sobre la idea original.

El bloque de enseñanza diseñado comprende un conjunto de tareas prácticas que desarrolla, simultáneamente, dos clases de contenido educativo: primero, el 'contenido táctico' (presente en la naturaleza común del juego motriz); y segundo, el contenido 'conflictivo colateral' (elemento de análisis crucial) englobado en la didáctica positiva del conflicto. Asimismo, aunque cada sesión está enlazada con un objetivo concreto, se remarca que el contenido presenta un carácter permanente y sumativo. De esta manera, se requiere que el propio docente ofrezca recuperación y fortalezca la memorización del estudiantado durante su proceso de trabajo. Para ello, empleará diferentes recursos didácticos: humanos (profesorado, alumnado, personal experto y/o modélico); materiales (fotografías, pósteres, esquemas, imágenes o titulares de periódicos y revistas); y tecnológicos (vídeos, audios, películas).

Paralelamente, el docente posibilita retroalimentación al grupo de estudiantes: primero, mostrando 'retroalimentación positiva' a las respuestas adecuadas del alumnado, verbalizando los refuerzos y difundiendo el conocimiento al grupo; segundo, aportando 'retroalimentación de recuerdo' a las respuestas desacertadasdefectuosas para permitir mayor comprensión y provocar nuevas propuestas de acción. No hay que olvidar el apoyo del grupo de iguales durante el proceso de aprendizaje: clarificando conocimientos, aportando procedimientos alternativos, buscando soluciones compartidas o actuando como orientador-guía.

Sobre la práctica educativa, cabe reflejar que toma su origen de la enseñanza comprensiva y se fundamenta sobre un enfoque organizativamente participativocolaborativo. De igual modo, el profesorado prioriza la reflexión, el diálogo y la negociación entre sus participantes. Además, los organiza en grupos mixtos y favorece la coeducación. Al tiempo que los agrupa desde la heterogeneidad en cuanto al nivel competencial o rendimiento motriz.

Respecto a la dinámica del juego, se le ofrece a cada equipo la posibilidad de encontrar respuestas a los 'problemas táctico-motrices' expuestos (mediante 'decisiones dialogadas'). Para ello, el docente diversifica el procedimiento del propio juego (con intención de modificar la implicación cognitiva) a través de situaciones cambiantes de ataque-defensa: (a) proponiendo variadas formas de conseguir puntos o resultados; (b) reduciendo o incrementando el número de jugadores (así como la reducción o ampliación de espacios libres); (c) añadiendo mayor número de balones para intensificar la complejidad en cuanto a los mecanismos de percepción y decisión; y (d) planteando la posibilidad de cambiar o crear nuevas reglas del juego (situaciones, todas ellas, que propician momentos de estrés y, consecuentemente, predisponen la aparición y/o escalada del conflicto).

Simultáneamente, cabe resaltar que el programa recoge diferentes estrategias de acción mediadora. Principalmente, el procedimiento gira en torno a coadyuvar a las partes a preparar y respetar sus encuentros, facilitando el diálogo y abriendo paso cuando se requiere. Asimismo, se trata de ofrecer espacios para que, con la mínima dependencia, se puedan posibilitar «encuentros privados» (con un tercero colaborador, normalmente un compañero); encuentros «entre las partes (sin la presencia de la persona mediadora)»; e incluso, «trabajando con una sola de las partes cuando la otra no desea participar» (Bolaños \& Urruela, 2013, p. 196).

El profesorado entrega tareas sistemáticas para que sus practicantes adquieran «la posibilidad de desarrollar habilidades comunicativas, autonomía, pensamiento crítico, capacidades de argumentación y proposición» (Aguirre-Loaiza \& Vanegas, 2011, p. 85).Y para conseguirlo, el educador trasciende el rol de mediador como «árbitro en la gestión del conflicto», para acercarse más a la de «dinamizador del proceso» (Ruiz, 2008, p. 97). Además, se planifican otros enfoques incorporados a los valores humanos del propio campo de la mediación: los «grupos cooperativos», el «círculo», el rincón del diálogo «boca-oreja» y la «asamblea de clase» (Boqué, 2018, pp. 92-95).

\section{Modelo didáctico}

A continuación, se muestra un ejemplo de sesiones del proceso formativo en el que quedan descritas, preferentemente, las intencionalidades del 'contenido conflictivo colateral' que se encuentran subordinadas al 'contenido táctico grupal' (tabla 3).

Finalmente, se presentan las especificaciones didácticas sobre la gestión del conflicto (tabla 4). 

riencia pedagógica, se recuerda la imposibilidad de establecer generalizaciones puesto que la investigación no se ha podido todavía completar. Como prospectiva, una vez aplicado el programa en su globalidad, se lleFuente: Elaboración propia

Tabla 4.

Indicaciones sobre cómo tratar el conflicto

\begin{tabular}{|c|c|c|c|c|}
\hline & \multicolumn{2}{|c|}{ ESPECIFICACIONES DIDACTICAS } \\
\hline & & & Rol del docente & Rol del alumnado \\
\hline \multirow{6}{*}{$\begin{array}{l}\text { MOMENTO DE LA } \\
\text { SESIÓN }\end{array}$} & $\begin{array}{l}\text { Antes de comenzar el } \\
\text { juego }\end{array}$ & $\begin{array}{l}\text { Puesta en marcha. } \\
\text { Orientaciones }\end{array}$ & $\begin{array}{l}\text {-Introducir (o recordar) las habilidades y reglas del diálogo } \\
\text { para cualquier momento de la sesión (cómo comunicarse } \\
\text { emisor y receptor para evitar malentendidos). } \\
\text {-Contemplar la naturalidad de las situaciones conflictivas y la } \\
\text { importancia de acogerlas con actitud de cambio y progreso. } \\
\text {-Utilizar un estilo constructivo para comunicarse con el } \\
\text { alumnado: planteando preguntas y dilemas para resolver } \\
\text { problemas (individual o en grupo). } \\
\text {-Implicarse como facilitador de diálogo y consultor del } \\
\text { proceso. }\end{array}$ & $\begin{array}{l}\text {-Expresar los sentimientos personalmente y de forma } \\
\text { clara (sin ser agresivo ni pasivo). } \\
\text {-Mantenerse receptivo a las necesidades de comunicación } \\
\text { de sus compañeros y adversarios de juego: empleando la } \\
\text { escucha activa (preguntas, aclaraciones, paráfrasis), sin } \\
\text { hacer juicios y sin intentar adivinar las intenciones del } \\
\text { otro. } \\
\text {-Ajustarse y respetar el carácter de sus diferentes } \\
\text { compañeros, aunque haciendo peticiones concretas para } \\
\text { buscar cambios conductuales. }\end{array}$ \\
\hline & \multirow{4}{*}{ Durante el juego } & $\begin{array}{l}\text { Cuando aparece } \\
\text { el enfado }\end{array}$ & $\begin{array}{l}\text {-Incapacitar formas erróneas de comunicación: usar mensajes } \\
\text { “yo”. } \\
\text {-Dar la oportunidad de visibilizar la frustración. } \\
\text { - Rebajar el desequilibrio de poder: hacer cumplir tiempos de } \\
\text { habla, separar problemas-personas. } \\
\text {-Ofrecer comprensión sobre el “ciclo vital” del enfado. }\end{array}$ & $\begin{array}{l}\text {-Aplicar cuatro pasos: } \\
\text { 1.Detenerse y serenarse fuera del juego. } \\
\text { 2.Identificar porqué se está furioso. } \\
\text { 3.Preguntarse a sí mismo si la ira está justificada con la } \\
\text { amenaza real. } \\
\text { 4.Decidir una acción para minimizar o eliminar la } \\
\text { amenaza. }\end{array}$ \\
\hline & & $\begin{array}{l}\text { Cuando se presenta } \\
\text { una disputa }\end{array}$ & $\begin{array}{l}\text {-Controlar los arrebatos de ira y su inadecuada reacción: } \\
\text { propiciar espacios y momentos para el cambio de percepción y } \\
\text { pensamiento. } \\
\text {-Hacer entrever la importancia de la colaboración como } \\
\text { actitud de diálogo. } \\
\text {-Poner en duda la relación entre la emoción sentida y la acción } \\
\text { realizada. }\end{array}$ & $\begin{array}{l}\text {-Decidir si se quiere hacer uso del 'Rincón del diálogo' } \\
\text { para establecer una negociación con el adversario. } \\
\text {-Seis pasos para la negociación: } \\
\text { 1. Saber qué quiere cada uno. } \\
\text { 2.Expresar qué se quiere que haga el otro. } \\
\text { 3.Entender qué posición toma el otro. } \\
\text { 4. Realizar una propuesta de acuerdo. } \\
\text { 5.Plantear alternativas a la propuesta. } \\
\text { 6.Aceptación del acuerdo. } \\
\end{array}$ \\
\hline & & Cuando existe una queja & $\begin{array}{l}\text {-Plantear preguntas al alumnado para el intercambio de } \\
\text { respuestas en pequeño grupo. } \\
\text {-Estimular la discusión-consenso de nuevas reglas de juego, } \\
\text { creación de tácticas más idóneas. } \\
\text {-Focalizar la queja en una petición para el adversario o el } \\
\text { propio compañero. } \\
\text {-Ayudar a los grupos antagónicos a trabajar juntos. }\end{array}$ & $\begin{array}{l}\text {-Comunicar percepciones y expresar sugerencias sin } \\
\text { culpabilizar al otro, sin gritar. } \\
\text {-Proponer al tomador de la queja una posible negociación } \\
\text { (yo estaría dispuesto a..., si tú estuvieras dispuesto a...). } \\
\text {-Centrarse en conseguir ganancias para todos, atacando a } \\
\text { la propuesta y no al negociador. }\end{array}$ \\
\hline & & $\begin{array}{l}\text { Cuando alguien } \\
\text { arremete contra otro }\end{array}$ & $\begin{array}{l}\text {-Determinar distanciamiento al agresor y prestar apoyo al } \\
\text { perjudicado. } \\
\text {-Recoger datos y necesidades; posibilitar y animar al diálogo } \\
\text { (pedagogía restaurativa). } \\
\text {-Apoyar, liberar tensiones y aproximar los malentendidos. } \\
\text {-Ayudar a los individuos o grupos antagónicos a trabajar } \\
\text { juntos. } \\
\text {-Incluir nuevas propuestas de aprendizaje que impliquen } \\
\text { positivamente a las partes en conflicto. }\end{array}$ & $\begin{array}{l}\text {-Aprender a parar, a identificar la causa de su acción y } \\
\text { generar un plan de respuesta compensadora (para } \\
\text { reeducar su mala acción y reparar el mal ocasionado). } \\
\text { - Aprender a controlar las emociones: analizar los posibles } \\
\text { pensamientos que hacen sentirse amenazado. } \\
\text {-Encontrar otras formas positivas de expresar cómo se } \\
\text { siente. }\end{array}$ \\
\hline & Al finalizar el juego & Autoevaluación Coevaluación & $\begin{array}{l}\text {-Actuar como moderador de debates y posibilitar a todo el } \\
\text { grupo ser 'generador de ideas'. } \\
\text {-Ofrecer un tiempo para establecer coevaluaciones entre los } \\
\text { equipos enfrentados en los juegos, o entre los jugadores de un } \\
\text { mismo equipo (de modo escrito y oral). } \\
\text {-Posibilitar un seguimiento (o regulación) de los conflictos } \\
\text { ocasionados anteriormente: en espacios como el Círculo (en la } \\
\text { propia pista de juego), la Asamblea (en la clase), o la Mediación } \\
\text { informal si fuera necesaria y apropiada (fuera del tiempo de la } \\
\text { sesión y con el maestro como facilitador). }\end{array}$ & $\begin{array}{l}\text {-Tomar parte y organizar la evaluación colaborativa: } \\
\text { analizar lo que se ha ofrecido en relación a las necesidades } \\
\text { del grupo; argumentar lo que puede o no servir; } \\
\text { reflexionar sobre las alternativas para que todos salgan } \\
\text { ganando; etc. } \\
\text {-Participar realizando autoevaluaciones o coevaluaciones } \\
\text { para posibilitar un intercambio de comprensión y una } \\
\text { retroalimentación a nivel de toda la clase. } \\
\text {-Comprender que la colaboración es necesaria para } \\
\text { mejorar cualquier situación de conflicto. }\end{array}$ \\
\hline
\end{tabular}

\section{Conclusiones}

A modo de conclusión, desde el ámbito del juego deportivo escolar, sostenemos que el método comprensivo-táctico podría ser una alternativa para educar la gestión positiva del conflicto, posibilitando que el alumnado enfrentara las barreras de la comunicación y vará a cabo un análisis de los resultados y se estimará el posible impacto en la convivencia escolar. Nuestro propósito final es enseñar competencias de resolución de conflictos en EF, pero que, a su vez, puedan trasladarse a otras materias académicas e incluso a otros ámbitos sociales, por lo que será fundamental trabajar desde un enfoque multidisciplinar y sumamente integrado. 


\section{Referencias}

Adigüzel, Ý. B. (2015). Peer Mediation in Schools. Procedia - Social and Behavioral Sciences, 174, 826-829. https://doi.org/http://dx.doi.org/10.1016/ j.sbspro.2015.01.674

Aguilar Párraga, M.A., \& Castañón Octavio, N. (2013). Una visión sobre la educación para la paz. Almanaque, (3), 69-89. Recuperado de http:// openjournal.unimet.edu.ve/index.php/Almanaque/article/view/34

Aguirre-Loaiza, H. H., \& Vanegas Forero, L. C. (2011). El conflicto: una alternativa de formación en la clase de educación física. Lúdica Pedagógica, 2(16), 81-86. https://doi.org/http://dx.doi.org/10.17227/ ludica.num16-1363

Alcaraz-Muñoz, V., Alonso Roque, J. I., \& Yuste Lucas, J. L. (2017). Jugar en positivo: género y emociones en educación física. Apunts. Educación Física y Deportes, 3er trimes(129), 51-63. https://doi.org/http: / / dx.doi.org/10.5672/apunts.2014-0983.es.(2017/ 2). 129.04

Ardila Roa, J. A., Jaimes Jaimes, G., Noy Martínez, M. O., Reina Cortés, B. O., \& Martínez Martínez, M. Y. (2019). La convivencia escolar a través de la educación física. Revista digital: Actividad Física y Deporte, 5(2), 16-39. https://doi.org/http://dx.doi.org/ 10.31910/rdafd.v5.n2.2019.1251

Aubert, A., Duque, E., Fisas, M., \& Valls, R. (2004). Dialogar y transformar. Padagogía crítica del siglo XXI (Primera Ed). Barcelona: Graó.

Barruel, C. A., \& Nissanka, M. (2018). Examining the Drivers of Success in the Peace Foundation Aotearoa/New Zealand PeerMediation Programs. En M. Walid Lutfy \& C. Toffolo (Eds.), Handbook of Research on Promoting Peace Through Practice, Academia, and the Arts (pp. 99-120). Hershey, United States: IGI Global.

Bello-Dávila, Z., Rionda-Sánchez, H. D., \& RodríguezPérez, M. E. (2010). La inteligencia emocional y su educación. VARONA, Revista Cientifico-Metodológica, 51, 36-43. Recuperado de http://www.redalyc.org/ articulo.oa?id $=360635569006$

Bercovitch, J., Kremenyuk, V., \& Zartman, W. (2009). Introduction: The Nature of Conflict and conflict Resolution. En J. Bercovitch, V. Kremenyuk, \& W. Zartman (Eds.), The SAGE Handbook of Conflict Resolution (pp. 1-11). London: SAGE Publications Ltd.

Bolaños Cartujo, I., \& Urruela Arnal, I. (2013). Una mirada aplicada: los espacios relacionales de la me- diación. Política y Sociedad, 50(1), 195-214. Recuperado de https://revistas.ucm.es/index.php/ $\mathrm{POSO} /$ article/view/39345

Boqué Torremorell, M. C. (2003). Cultura de mediación y cambio social (Primera Ed). Barcelona: Gedisa Editorial.

Boqué Torremorell, M. C. (2007). Prevención de conflictos y mediación escolar. Idea-La Mancha:Revista de Educación, (4), 60-66. Recuperado de https:// dialnet.unirioja.es/servlet/ articulo? codigo $=2506060$

Boqué Torremorell, M. C. (2018). La mediacion va a la escuela. Hacia un buen plan de convivencia en el centro. Madrid: Narcea.

Boqué Torremorell, Maria Carme. (2020). Prácticas restaurativas para la prevención y gestión de los conflictos. 20 Círculos de la palabra y una Asamblea en el aula. Madrid: Narcea.

Burton, J.W. (1990). Conflict:Human NeedsTheory. London: The Macmillan Press.

Burton, J. W. (1997). Conflict Resolution: Towards Problem Solving. Peace and Conflict Studies, 4(2. Article 2), 5. Recuperado de https:// nsuworks.nova.edu/pcs/vol4/iss2/2/

Buscà, F., Ruiz, L., \& Rekalde, I. (2015). Tratamiento del conflicto en las Comunidades de Aprendizaje a través de la educación física. Retos. Nuevas tendencias en Educación Física, Deporte y Recreación, 25, 156-161. https://doi.org/https://doi.org/10.47197/ retos.v0i25.34503

Canales Nuñez, P., Aravena Kenigs, O., CarcamoOyarzun, J., Lorca Tapia, J., \& Martinez-Salazar, C. (2018). Prácticas pedagógicas que favorecen u obstaculizan la inclusión educativa en el aula de educación física desde la perspectiva del alumnado y profesorado. Retos, 34, 212-217. https://doi.org/ 10.47197/retos.v0i34.59620

Cantón Chirivella, E., \& Leon Zarceno, E. (2005). La resolución de conflictos en la práctica deportiva escolar. Cuadernos de Psicología del Deporte, Vol. 5, pp. 159-171. https://revistas.um.es/cpd/article/ view/93471

Cantos, J., \& Moreno, F. J. (2019). Pedagogía no lineal como método de enseñanza de los comportamientos tácticos en los deportes de equipo, aplicación al rugby. Retos, 35, 402-406. https://doi.org/ 10.47197/retos.v0i35.63508

Capllonch Bujosa, M., Figueras Comas, S., \& Castro, M. (2018). Estrategias para la resolución de conflictos en educación física. Apunts. Educación física y depor- 
tes, 3(133), 50-67. https://doi.org/http:// dx.doi.org/10.5672/apunts.2014-0983.es.(2018/ 3). 133.04

Capllonch Bujosa, M., Figueras Comas, S., \& Lleixà Arribas, T. (2015). Prevención y resolución de conflictos en educación física: estado de la cuestión. Retos: nuevas tendencias en educación física, deporte y recreación, (25), 149-155. https://doi.org/https:// doi.org/10.47197/retos.v0i25.34502

Castejón Oliva, F., \& Díaz del Cueto, M. (2011). La enseñanza comprensiva del deporte: dificultades del profesorado en el diseño de tareas y en la estrategia pregunta-respuesta. Tandem: didactica de la educacion fisica. Recuperado de https://repositorio.uam.es/ handle/10486/665959

Cebolla-Baldoví, R. (2021). Aprender a gestionar conflictos en Educación Física: Diseño de un programa integrado de mejora de la convivencia [Tesis de doctorado no publicada]. Universidad de Valencia.

Cepero González, M., Marín Regalado, M. N., \& Torres Guerrero, J. (2010). Teaching and learning social values: experience of resolution of conflicts in the classroom of physical education across the learning of social skills. Journal of Human Sport and Exercise, 5(3), 497-506. https://doi.org/http:// dx.doi.org/10.4100/jhse.2010.53.20

Condette, S. (2020). The Contribution of Peer Mediation to the Implementation of Human Rights Education. En J. Zajda (Ed.), Human Rights Education Globally. Globalisation, Comparative Education and Policy Research (pp. 159-172). Recuperado de https:// link.springer.com/chapter/10.1007\%2F978-94024-1913-9_8\#citeas

Delors, J. (1996). La educacion encierra un tesoro. Madrid: Santillana. Ediciones Unesco.

Devís-Devís, J. (1996). Educación física, deporte y currículum. Investigación y desarrollo curricular. Madrid: Aprendizaje Visor.

Dummer, S. R. (2010). Peer mediation: What school counselors need to know. Recuperado de https:// www. semanticscholar.org / paper / PeerMediation\%3A-What-School-Counselors-Need-to-

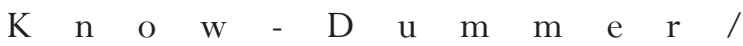
c67f2470cab8ed9e20a7f29b551e84ef1b7f4f55

Flecha García, R., \& García Yeste, C. (2007). Prevención de conflictos en las comunidades de aprendizaje. Idea La Mancha: Revista de Educación, (4), 72-76. Recuperado de https: / / dialnet.unirioja.es/servlet/ articulo?codigo $=2506069$

Funes Lapponi, S. (2000). Resolución de conflictos en la escuela: una herramienta para la cultura de paz y la convivencia. Contextos Educativos. Revista de Educación, (3), 91-106. Recuperado de https:// publicaciones.unirioja.es/ojs/index.php/contextos/ article/view/466

Fuquen Alvarado, M. E. (2003). Los conflictos y las formas alternativas de resolución. Tabula Rasa, (1), 265278. Recuperado de https: / /www.redalyc.org/pdf/ 396/39600114.pdf

García-Raga, L., Boqué Torremorell, M. C., \& Grau Vidal, R. (2019). Valoración de la mediación escolar a partir de la opinión de alumnado de educación secundaria de Castellón, Valencia y Alicante (España). Profesorado. Revista de Currículum y Formación del Profesorado, 23(1), 103-119. https://doi.org/http:// dx.doi.org/10.30827/profesorado.v23i1.9146

García-Raga, L., \& López-Martín, R. (2010). La convivencia escolar: una mirada pedagógica, política y prospectiva. Universidad de Valencia.

Gellin, M. (2018). Mediation in Finnish Schools: From Conflicts to Restoration. En A. Nylund, K. Ervasti, \& L.Adrian (Eds.), Nordic Mediation Research (pp. 247266). Recuperado dehttps://doi.org/10.1007/9783-319-73019-6_13

Hamilton, M. (2015). A Pedagogy for Peacebuilding: Practicing an Integrative Model for Conflict Analysis and Response. Revista de Mediación, 8(2. e3), 9. Recuperado de https://revistademediacion.com/ articulos/una-pedagogia-la-consolidacion-la-pazpractica-modelo-integrador-analisis-la-respuesta-losconflictos/

Harris, R. D. (2005). Unlocking the learning potential in peer mediation: An evaluation of peer mediator modeling and disputant learning. Conflict Resolution Quarterly, 23(2), 141-164. Recuperado de https:// doi.org/10.1002/crq.130

Hernández Mite, K. D., Morán Franco, M. R., \& Bucheli Bermúdez, B. A. (2018). La actividad física y el desarrollo emocional: una propuesta a considerar en la práctica educativa. Revista Conrado, 14(63), 159-163. Recuperado de https://conrado.ucf.edu.cu/ index.php/conrado/article/view/772

Hocker, J. L., \& Wilmot, W. W. (1985). Interpersonal conflict (Segunda Ed). Estados Unidos de América: W.C. Brown Co.

Ibarrola-García, S., \& Iriarte Redín, C. (2013). Percepción de mejora personal y social en los alumnos mediadores y mediados y profesores mediadores. Campo abierto, 32(2), 117-143. Recuperado de https: / / dialnet.unirioja.es/servlet/ 
articulo?codigo $=4838109$

Iglesias Ortuño, E., \& Ortuño Muñoz, E. (2018). Trabajo Social y mediación para la convivencia y el bienestar escolar. Cuadernos de Trabajo Social, 31 (2), 381 392. https: / /doi.org/http:/ /dx.doi.org/10.5209/ CUTS. 53374

Jaqueira, A. R., Lavega-Burgues, P., Lagardera Otero, F., Aráujo, P., \& Rodrigues, M. (2014). Educando para la paz jugando: género y emociones en la práctica de juegos cooperativos competitivos. Educatio Siglo XXI, 32(2), 15-32. https://doi.org/http: / / dx.doi.org/10.6018/j/194071

Jares, X. R. (1997). El lugar del conflicto en la organización escolar. Revista Iberoamericana de Educación, (15), 53-73. Recuperado de http:// www.comitepaz.org.br/download/El lugar del conflicto en la organización escolar.pdf

Jares, X. R. (2001). Educacion y conflicto: Guia de educacion para la convivencia (Segunda ed). Madrid: Editorial Popular.

Leiva Olivencia, J. J. (2008). Interculturalidad, gestión de la convivencia y diversidad cultural en la escuela: un estudio de las actitudes del profesorado. Revista Iberoamericana de Educación, 46(2), 1-14. Recuperado de https:/ / rieoei.org/RIE/article/view/2003

Leiva Olivencia, J. J. (2011). Educación y conflicto en escuelas inclusivas: Principios, valores y estrategias para mejora de la convivencia escolar. En J. J. Maquilón Sánchez, A. B. Mirete Ruiz, A. Escarbajal Frutos, \& A. M. Giménez Gualdo (Eds.), Cambios educativos y formativos para el desarrollo humano y sostenible (pp. 371-378). Murcia: Universidad de Murcia, Servicio de Publicaciones.

López-Ros, V., Castejón-Oliva, F. J., Bouthier, D., \& Llobet-Martí, B. (2015). Modelos para una enseñanza comprensiva del deporte. Espacios comunes para el encuentro (y algún desencuentro). Agora para la Educación Física y el Deporte, 17(1), 45-60. Recuperado de https: / / repositorio.uam.es/handle/10486/ 670820

Martín-Barrero, A., \& Camacho Lazarraga, P. (2020). El diseño de tareas de entrenamiento en el fútbol desde el enfoque de la pedagogía no lineal. Retos, 38, 768-772. https://doi.org/10.47197/ retos.v38i38.76612

Martín Hocajo, M., \& Ríos González, O. (2015). Prevención y resolución del conflicto en educación física desde la perspectiva del alumnado. Retos: nuevas tendencias en Educacion Física, Deporte y Recreacion, (25), 162-167. https://doi.org/https://doi.org/ 10.47197/retos.v0i25.34506

Martínez Usarralde, M. J., Sahuquillo Mateo, P., \& García-Raga, L. (2013). Identidad y responsabilidades socioeducativas del mediador escolar y del mediador intercultural: hacia una clarificación de funciones. Mediaciones Sociales, (11), 56-81. https:// doi.org/http://dx.doi.org/10.5209/ rev_MESO.2012.v11.41269

Medina Cascales, J. Á., \& Reverte Prieto, M. J. (2019). Incidencia de la práctica de actividad física y deportiva como reguladora de la violencia escolar. Retos, 35, 54-60. https://doi.org/10.47197/ retos.v0i35.64359

Miranzo de Mateo, S. (2018). INSERT modelo de mediación escolar para el desarrollo de competencias sociocognitivas, emocionales y morales. Padres y Maestros / Journal of Parents and Teachers, (373), 6-14. https: / /doi.org/http://dx.doi.org/10.14422/ pym.i373.y2018.001

Monzonís Martínez, N., \& Capllonch Bujosa, M. (2014). La educación física en la consecución de la competencia social y ciudadana. Retos: nuevas tendencias en educación física, deporte y recreación, (25), 180-185. Recuperado de http: / dialnet.unirioja.es/servlet/ oaiart? codigo $=4555277$

Munduate, L., Ganaza, J., \& Alcaide, M. (1993). Estilos de gestión del conflicto interpersonal en las organizaciones. Revista de Psicología Social, 8(1), 47-68. https: / doi.org/10.1080/02134748.1993.10821669

Ortega-Ruiz, R., \& Del Rey Alamillo, R. (2006). La mediación escolar en el marco de la construcción de la convivencia y la prevención de la violencia. Avances en supervisión educativa, 2, 1-7. Recuperado de https: / /idus.us.es/xmlui/handle/11441/59340

Pinto Da Costa, E., \& Oliveira Sá, S. (2020). Conflict Mediation at School: Literature Review with webQDA®. En A. P. Costa, L. P. Reis, \& A. Moreira (Eds.), Computer Supported Qualitative Research.WCQR 2019. Advances in Intelligent Systems and Computing (pp. 114-123). Recuperado de https: / /doi.org/10.1007/ 978-3-030-31787-4_9\%0A

Rozemblum de Horowitz, S. (1998). Mediación en la escuela. Resolución de conflictos en el ámbito educativo adolescente (Primera Ed; Aique, Ed.). Buenos Aires.

Ruiz Omeñaca, J. V. (2008). Educación física, valores éticos y resolución de conflictos: reflexiones y propuestas de acción. En A. Fraile Aranda (Ed.), La resolución de los conflictos en y a través de la educación física (pp. 65-116). Barcelona: Graó.

Sáez de Ocáriz Granja, U. (2011). Conflictos y educa- 
ción física a la luz de la praxiología motriz. Estudio de caso de un centro educativo de primaria. TDX (Tesis Doctorals en Xarxa). Recuperado de http:// www.tesisenred.net/handle/10803/53637

Sáez de Ocáriz Granja, U., \& Lavega-Burgues, P. (2014). Hacia una transformación de los conflictos motores en educación física. Cultura, Ciencia y Deporte, 9(25), 43-55. Recuperado de https://ccd.ucam.edu/ index.php/revista/article/view/388

Sáez de Ocáriz Granja, U., \& Lavega-Burgués, P. (2015). Estudio de los conflictos en el juego en educación física en primaria. Revista Internacional de Medicina y Ciencias de la Actividad Física y del Deporte, (57), 29-44. https://doi.org/http://dx.doi.org/10.15366/ rimcafd2015.57.003

Sáez de Ocáriz Granja, U., Lavega-Burgués, P., Lagardera Otero, F., Costes Rodríguez, A., \& Serna Berdavío, J. (2014). ¿Por qué te peleas? Conflictos motores y emociones negativas en la clase de Educación Física/ : el caso de los juegos de oposición. Educatio Siglo XXI, 32(1), 71-90. https://doi.org/ http://dx.doi.org/10.6018/j/194091

Sáez de Ocáriz Granja, U., Lavega-Burgues, P., \& March-Janés, J. (2013). El profesorado ante los conflictos en la educación física. El caso de los juegos de oposición en Primaria. Revista Electronica Interuniversitaria de Formación del Profesorado, 16(1), 163176. https://doi.org/http://dx.doi.org/10.6018/ reifop.16.1.180101

Samy Hassan, E. (2019). The Effectiveness of Peer Mediation on Students' Discipline Referral Rates at Al Yasat Middle School. Journal of Innovation and Creativity Research, 1(2), 83-93. Recuperado de http:/ /www.jicr.net/images/Vol1_iss_2_2019/ AR12_Hassan_2019.pdf

Sánchez-Gómez, R., Devís-Devís, J., \& Navarro-Adelantado, V. (2014). El modelo Teaching Games for Understanding en el contexto internacional y español: una perspectiva histórica. Ágora para la educación física y el deporte, 3(16), 197-213. Recuperado de https: / / dialnet.unirioja.es / servlet/ articulo?codigo $=4908241$

Sánchez Fernández, S. (2018). La convivencia escolar desde la perspectiva de investigación para la cultura de paz. Participación educativa, 5(8), 55-68. Recuperado de https://dialnet.unirioja.es/servlet/ articulo?codigo $=6785341$

Sanchez Garcia-Arista, M. L. (2016). Mediación educativa contextualizada. En M. L. Sánchez García-Arista (Ed.), Gestion positiva de conflictos y mediacion en contextos educativos (Segunda Ed, pp. 19-43). Madrid: Reus.

Silva Lorente, I., \& Torrego Seijo, J. C. (2017). Percepción del alumnado y profesorado sobre un programa de mediación entre iguales. Qualitative Research in Education, 6(2), 214-238. Recuperado de https:// hipatiapress.com/hpjournals/index.php/qre/ article/view/2713

Torrego Seijo, J. C. (2006). Modelo integrado de mejora de la convivencia. Estrategias de mediación y tratamiento de conflictos (Primera Ed). Barcelona: Graó. 227.

Torrego Seijo, J. C. (2013). La ayuda entre iguales para mejorar la convivencia escolar: Manual para la formacion de alumnos ayudantes. Madrid: Narcea.

Tuvilla Rayo, J. (2004). Cultura de paz: fundamentos y claves educativas. Bilbao: Ed. Desclée De Brouwer.

Velázquez-Callado, C. (2008). Las actividades cooperativas como recurso para el tratamiento de los conflictos en las clases de educación física. En La resolución de los conflictos en y a través de la educación física (Primera Ed, pp. 117-161). Barcelona: Graó.

Viana Orta, M. I., \& López Francés, I. (2015). Aportación de la mediación escolar a la igualdad y a la inclusión social. Revista nacional e internacional de educación inclusiva, 8(1), 14-26. Recuperado de https:// revistaeducacioninclusiva.es/index.php/REI/ article/view/118

Villalba Cano, J. (2016). Prácticas curriculares para una convivencia y cultura de paz positiva. Itinerario Educativo, 30(68), 131-146. Recuperado de http:// revistas.usbbog.edu.co/index.php/Itinerario/ article/view/2952

Whiteside, R. (2016). Peer Mediation as aViable Option for School Conflict Resolution Programs. Undergraduate Journal of Service Learning and Community-Based Research, 5, 1-10. Recuperado de https: / / ujslcbr.org/index.php/ujslcbr/article/ view/237

Zañartu Correa, L. M. (2003). Aprendizaje colaborativo: una nueva forma de Diálogo Interpersonal y en Red. Revista Digital de Educación y Nuevas Tecnologías, (28), 1-9. Recuperado de http://files.enriquecereducaciontic.webnode.es/200000026-9a1009c069/ C o n t e x t o _ E d u c a t i v o __Revista_digital_de_Educacion_y_Nuevas_Tecnologias.pdf 